\title{
Stress Urinary Incontinence Surgery Trends in Academic Female Pelvic Medicine and Reconstructive Surgery Urology Practice in the Setting of the Food and Drug Administration Public Health Notifications
}

\author{
Goran Rac, ${ }_{1}^{1}$ Austin Younger, ${ }^{1}$ James O. Clemens, ${ }^{2}$ Kathleen Kobashi, ${ }^{3}$ Aqsa Khan, ${ }^{4}$ Victor Nitti, ${ }^{4}$ Ilana Jacobs, ${ }^{5}$ \\ Gary E. Lemack, ${ }^{5}$ Elizabeth T. Brown, ${ }^{6}$ Roger Dmochowski, ${ }^{6}$ Lara MacLachlan, ${ }^{7}$ Arthur Mourtzinos, ${ }^{7}$ \\ David Ginsberg, ${ }^{8}$ Michelle Koski, ${ }^{9}$ Ross Rames, ${ }^{1}$ and Eric S. Rovner ${ }^{1 *}$ \\ ${ }^{1}$ Department of Urology, Medical University of South Carolina, Charleston, South Carolina \\ ${ }^{2}$ Department of Urology, University of Michigan Health Science Center, Ann Arbor, Michigan \\ ${ }^{3}$ Section of Urology and Renal Transplantation, Virginia Mason Medical Center, Seattle, Washington \\ ${ }^{4}$ Department of Urology, New York University Langone Medical Center, New York, New York \\ ${ }^{5}$ Department of Urology, University of Texas Southwestern Medical Center, Dallas, Texas \\ ${ }^{6}$ Department of Urologic Surgery, Vanderbilt University Medical Center, Nashville, Tennessee \\ ${ }^{7}$ Institute of Urology, Lahey Hospital and Medical Center, Burlington, Massachusetts \\ ${ }^{8}$ Institute of Urology, Keck Medicine of University of Southern California, Los Angeles, California \\ ${ }^{9}$ Urology of Kaiser Permanente Medical Center, San Diego, California
}

\begin{abstract}
Aims: To investigate the possible effects of the Food and Drug Administration (FDA) Public Health Notifications in 2008 and 2011 regarding surgical trends in transvaginal mesh (TVM) placement for stress urinary incontinence (SUI) and related mesh revision surgery in Female Pelvic Medicine \& Reconstructive Surgery (FPMRS) practice in tertiary care academic medical centers in the United States. Methods: Surgical volume for procedures performed primarily by FPMRS surgeons at eight academic institutions across the US was collected using Current Procedural Terminology (CPT) codes for stress urinary incontinence repair and revision surgeries from 2007 to 2013 . SAS statistical software was used to assess for trends in the data. Results: There was a decrease in the use of synthetic mesh sling for the treatment of SUI at academic tertiary care centers over the past 7 years; however, this was not statistically significant. While the total number of surgical interventions for SUI remained stable, there was an increase in the utilization of autologous fascia pubovaginal slings (AFPVS). The number of mesh sling revision surgeries, including urethrolysis and removal or revision of slings, increased almost three-fold at these centers. Conclusions: These observed trends suggest a possible effect of the FDA Public Health Notifications regarding TVM on surgical practice for SUI in academic centers, even though they did not specifically warn against the use of synthetic mesh for this indication. Indications for surgery, complications, and outcomes were not evaluated during this retrospective study. However, such data may provide alternative insights into reasons for the observed trends. Neurourol. Urodynam. 36:1155-1160, 2017. (c) 2016 Wiley Periodicals, Inc.
\end{abstract}

Key words: academic medical center; Food and Drug Administration; mesh; revision surgery; sling; stress urinary incontinence

\section{INTRODUCTION}

Synthetic midurethral mesh slings have become the gold standard in surgical repair of stress urinary incontinence (SUI) in the field of Female Pelvic Medicine and Reconstructive Surgery (FPMRS), replacing needle suspension and Burch colposuspension as the most popular procedure for SUI. ${ }^{1-3}$ However, there is increasing controversy surrounding the use of transvaginal mesh (TVM) to treat SUI and pelvic organ prolapse (POP). Synthetic mesh for use in SUI repair was first approved in 1998 by the Food and Drug Administration (FDA) via 510(k) Premarket Notification after finding it substantially equivalent to surgical mesh for hernia repair. ${ }^{4}$ In October of 2008, the FDA issued a Public Health Notification and Safety Communication due to concern over increased reporting through the Manufacturer and User Device Experience (MAUDE) database of complications associated with transvaginal placement of surgical mesh (TVM) to treat SUI and POP. ${ }^{5}$ This was followed in July of 2011 with an updated Safety Communication released by the FDA on the safety and efficacy of TVM for POP. ${ }^{6}$ Of note, this second July 2011 Public Health Notification did not comment specifically on the safety of using mesh for the treatment of SUI. Complications associated with midurethral mesh sling repair of SUI include pain, dyspareunia, mesh erosion and exposure. ${ }^{7}$

Dr. Mickey Karram led the peer-review process as the Associate Editor responsible for the paper.

Potential conflicts of interest: Dr. Rac, Dr. Younger, Dr. Clemens, Dr. Kobashi, Dr. Khan, Dr. Jacobs, Dr. Brown, Dr. MacLachlan, Dr. Ginsberg, Dr. Koski, Dr. Rames, and Dr. Rovner have nothing to disclose. Dr. Nitti reports that he is a Clinical Trials Investigator for Astellas, Allergan, and Cook MyoSite, outside the submitted work. Dr. Lemack reports personal fees from Astellas, Allergan, and Medtronic, outside the submitted work. Dr. Dmochowski reports being a Consult for Allergan and Medtronic, and personal fees from Allergan and Medtronic, outside the submitted work. Dr. Mourtzinos reports personal fees from Boston Scientific Corporation, Allergan, Astellas Pharmaceuticals, and Medtronic, Inc., outside the submitted work.

Grant sponsor: National Center for Advancing Translational Sciences; Grant number: UL1TR000062

*Correspondence to: Eric S. Rovner, M.D., Department of Urology, Medical University of South Carolina, 96 Jonathan Lucas St., CSB 644, Charleston, SC 29425. E-mail: rovnere@musc.edu

Received 6 April 2016; Accepted 5 July 2016

Published online 26 July 2016 in Wiley Online Library

(wileyonlinelibrary.com)

DOI 10.1002/nau.23080 
Interestingly, Rice et al. found that there has been an increase in patient perceived mesh complications since the 2011 Public Health Notification, but that the actual frequency, type, and location of mesh complications has not changed. ${ }^{8}$ Patient perceptions of vaginal mesh have been affected by a variety of factors in recent years, including advertisements for recruitment of plaintiffs for mesh litigation. ${ }^{9}$ Nevertheless, the effect of the FDA Safety Communications on surgical practice for SUI remains unclear. We recently showed that there has been a significant change in POP surgery in this practice setting over the last seven years, with a substantial reduction in transvaginal mesh augmented repair of POP as well as a simultaneous increase in the number of mesh revision surgeries. ${ }^{10}$ The objective of this study is to evaluate for changes in trends in SUI surgery, including sling revisions for mesh-related complications among FPMRS specialists over the last 7 years at tertiary care medical centers across the United States.

\section{MATERIALS AND METHODS}

Surgical volume of procedures performed by FPMRS specialists at eight academic institutions was collected using CPT codes for SUI sling procedures (57288), urethrolysis (53500), transvaginal sling incision (57287), and autologous fascia procedures $(20920,20922,20926)$ (Appendix 1). The study was approved by the Medical University of South Carolina institutional review board and was inclusive of all participating academic institutions. Only case volume information was exchanged between participating centers and all patient information was de-identified.

The eight tertiary referral centers participating in this study were: Medical University of South Carolina, Charleston, SC; University of Michigan Health Science Center, Ann Arbor, MI; Virginia Mason Medical Center, Seattle, WA; New York University Langone Medical Center, New York, NY; University of Texas Southwestern Medical Center, Dallas, TX; Vanderbilt University Medical Center, Nashville, TN; Lahey Hospital \& Medical Center, Burlington, MA; Keck Medicine of University of Southern California, Los Angeles, CA. These were chosen in order to represent a broad geographic range across the US and each section of the American Urologic Association. Each institution involved had at least one senior surgeon that was Board Certified in Urology, a member of Society of Urodynamics, Female Urology and Urogenital Reconstruction (SUFU), considered a specialist at their institution for Female Pelvic Medicine, and is presently board-certified in FPMRS (though that Board was not available during the entire time period that encompassed the CPT billing data collection).

The first FDA Public Health Notification addressing transvaginal mesh complications was released October 2008 with an updated statement in July 2011. To allow for a margin of over 1.5 years of practice stability prior to and after the notifications, a time period of January 1, 2007 to December 31, 2013 was selected to investigate the potential effect of the notifications on SUI surgical practice. A total of 7,739 surgical procedures related to SUI across the eight institutions during the study period were identified. These included both synthetic and fascial sling interventions, as well as sling revision surgeries. Two centers (University of Michigan Health Science Center and Virginia Mason Medical Center) were partially excluded from the analysis except for the evaluation of trend in urethral revision surgery due to variations in coding practice, in that we could not distinguish synthetic midurethral sling from autologous fascia pubovaginal sling (AFPVS) because they only used code 57288 and did not include separate CPT codes for graft harvesting. After exclusion of these cases, 4,788 surgical procedures were included across the eight institutions. The total number of mesh slings was calculated by subtracting the instances of CPT codes for autologous fascia grafts (20920, 29022, 20926) from the total number of instances of the CPT code for sling operation for stress incontinence (57288). The number of mesh revision surgeries was calculated by adding together the instances of CPT codes for urethrolysis (53500) and removal or revision of sling for stress incontinence (57287).

Statistical analysis was performed using SAS statistical software. The observed trends were assessed by determining Spearman correlation coefficients and performing Pearson's chi-squared tests when appropriate. Significance of trend was defined by $P$-value $<0.05$. The data was also stratified by academic center and analyzed with the aforementioned methods in order to assess for potential weighted bias or center effect.

\section{RESULTS}

This multi-institutional collaborative study included a total of 4,788 interventions for SUI performed between January 1 , 2007 and December 31, 2013. During the 7 years evaluated, a decrease in the use of synthetic mesh to treat SUI was noted (Fig. 1); however, this was not statistically significant $(P=0.25)$. Of note, two centers (New York University Langone Medical Center and Vanderbilt University Medical Center) did have a statistically significant decrease in the utilization of mesh for SUI during this time $(P=0.036$ and $P=0.016$, respectively). One center (Lahey Hospital \& Medical Center) had an overall increase in mesh implantation during this time period that was not statistically significant $(P=0.76)$. A subanalysis of the data looking at dates from 2011 to 2013 was done to evaluate for the possibility of an independent effect of the second FDA Public Health Notification from July 2011 on practice patterns. In this subanalysis, there was a significant decrease observed in use of synthetic mesh from 2011 to 2013. However, when this subanalysis was stratified by center, it was found to be largely the effect of practice patterns at Lahey Hospital \& Medical Center, which had a significant drop-off after a brief period of rapid increase from 2009 to 2010. The remaining centers did not have a significant change utilization of mesh since 2011.

Surgical volume for treatment of SUI has remained relatively stable over the past 7 years (Fig. 2); however, there has been a significant increase in the utilization of AFPVS when compared to total SUI intervention, from $21 \%$ of all SUI surgeries in 2007 to $30 \%$ in 2013 (Fig. 3, $P<0.0001$ ). Over this same time period, the number of urethral revision surgeries (urethrolysis and sling incision) increased by almost three-fold from 145 in 2007 to 392 in 2013. (Fig. 4, $P=0.00045$ ).

\section{DISCUSSION}

Current trends in surgical management of stress urinary incontinence have not been well defined in this era of increased scrutiny over procedures using synthetic vaginal mesh. Several studies have investigated these trends for both SUI and POP in the past. However, the majority of these featured end-points that do not include the FDA Public Health Notifications released in 2008 and 2011. As a part of the Urologic Diseases of America Project, Anger et al. (2009) randomly sampled Medicare data of female beneficiaries aged greater than 65 years to investigate patterns in the surgical treatment of women with SUI in the United States from 1992 


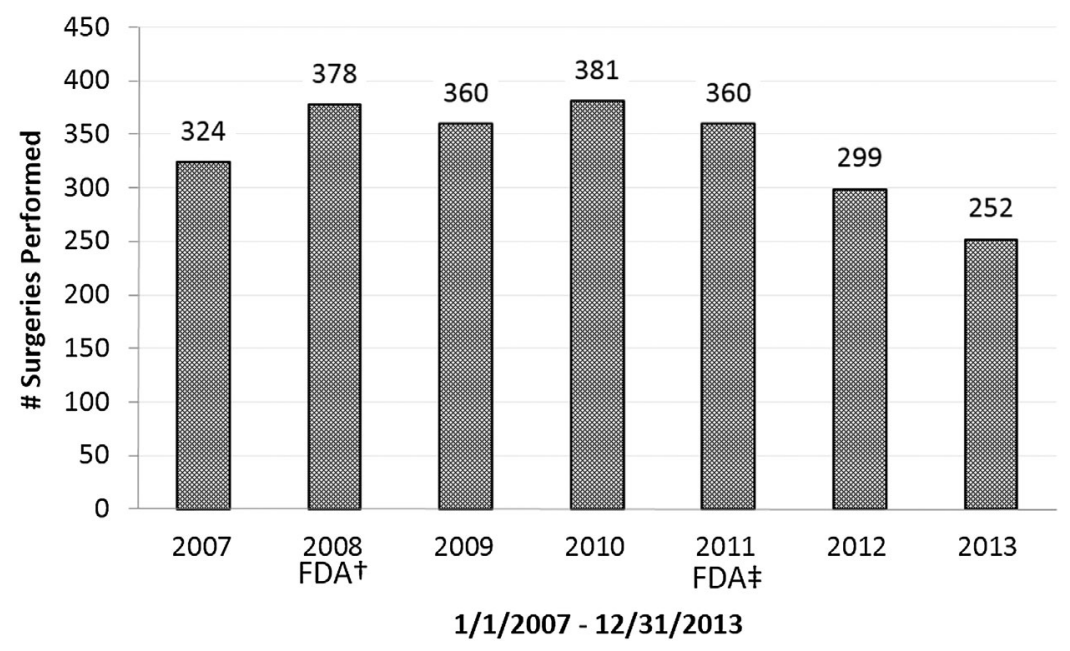

Fig. 1. Trend of mesh implant surgery for treatment of stress urinary incontinence surgery. Calculated by subtracting the instances of CPT codes that signify use of autologous fascia $(20920,29022,20926)$ from the total number of instances of the CPT code for sling operation for stress incontinence (57288). FDA $\dagger-$ October 2008 Public Health Notification and Safety Communication, FDA -July 2011 UPDATE to Public Health Notification and Safety Communication.

to $2001 .^{2}$ They found a "drastic increase" in the numbers and rates of sling placements for SUI from 1995 to 2001, gradually replacing needle suspension as the most commonly performed incontinence procedure. Rogo-Gupta et al. (2013) continued this study by looking at Medicare data from 2002 to $2007 .{ }^{11}$ They reported a continued increase in sling procedures performed each year. However, it is important to note that the authors of both studies were unable to differentiate between bladder neck fascial slings and midurethral synthetic slings, because they share the same CPT code (57288). It is likely that the majority of those procedures were midurethral synthetic slings; however, in our study, we were able to circumvent this potential limitation by subtracting the instances of CPT codes for autologous fascia harvest from the total instances of the CPT code 57288 to determine the number of procedures utilizing synthetic slings for most centers. Due to this methodology and depending on center coding practice, some centers were excluded from our data set which is a limitation of our study design. Another limitation of the studies by Anger et al. and Rogo-Gupta et al. is that Medicare patients over the age of 65 years reflect only a subset of women who suffer from SUI. Funk Johnson et al. published a similar study in 2012 looking at trends in SUI surgery between 2000 and 2009; however, their focus was on women between the ages of 18 and 64 years. ${ }^{3}$ They utilized the Thomson Reuters MarketScan Commercial Claims and Encounters database which contains healthcare claims data from employer-based plans in the United States. The authors noted increasing rates of sling procedures in this population over the years studied, with the greatest increase being in the group of women aged 35-50 years old when adjusted for age. This study was also limited by the lack of differentiation between bladder neck fascial slings and midurethral synthetic slings.

Another finding of the study by Rogo-Gupta et al. was an increasing trend in surgical management of SUI in ambulatory surgery centers, with a simultaneous decline in inpatient management. ${ }^{11}$ A 2012 study looking at trends in ambulatory procedures for SUI by Suskind et al. found a four-fold increase in midurethral sling procedures from 2001 to $2009 .{ }^{12}$ The authors

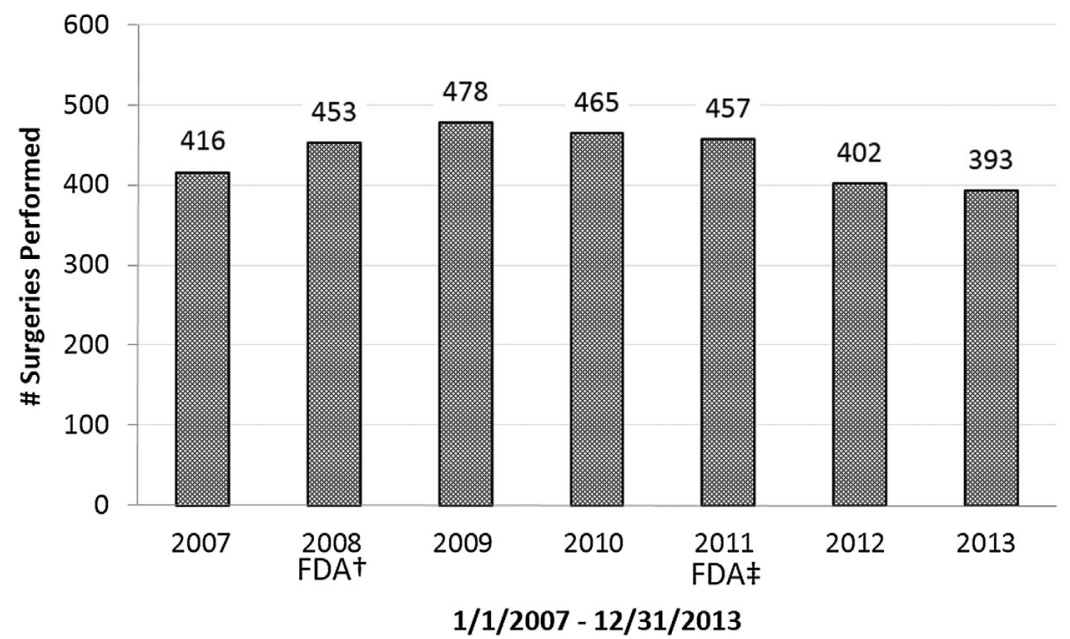

Fig. 2. Trend in surgical volume for stress urinary incontinence, not including revision surgery. CPT code 57288. FDA $\dagger-$ October 2008 Public Health Notification and Safety Communication, FDA 


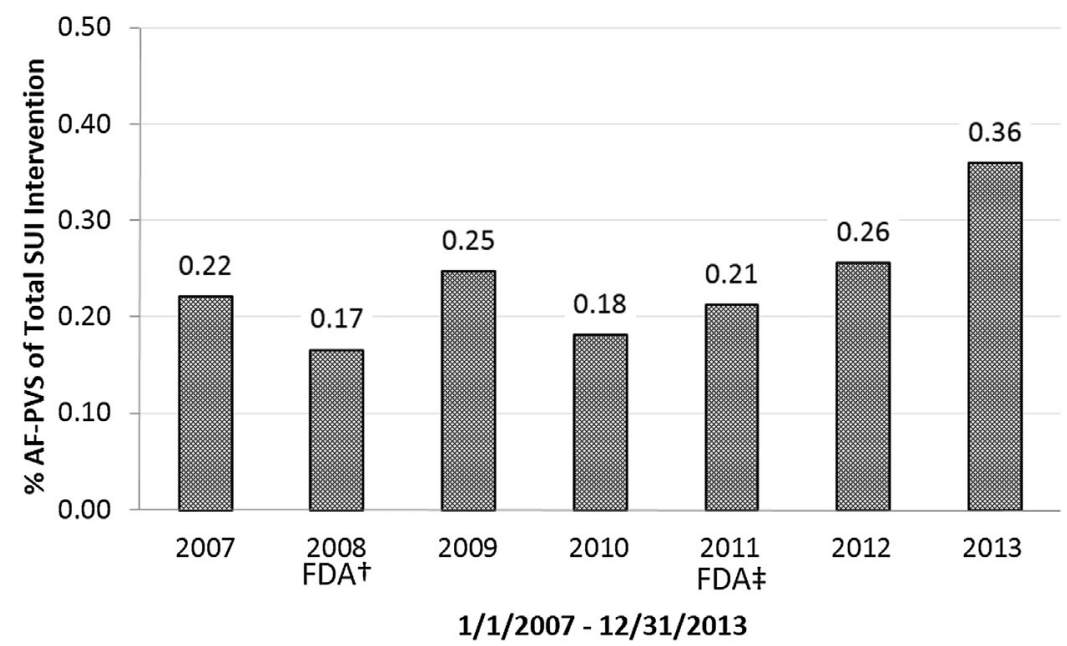

Fig. 3. Trend in autologous fascia pubovaginal sling procedures for surgical treatment of stress urinary incontinence. Calculated as a ratio of CPT codes that signify use of autologous fascia $(20920,29022,20926)$ over the CPT code for stress incontinence (57288). FDA $\uparrow-O c t o b e r ~ 2008$ Public Health Notification and Safety Communication, FDA $\$-J u l y ~ 2011$ UPDATE to Public Health Notification and Safety Communication.

believe that they were potentially able to mitigate the limitation of lack of a specific CPT code to specify sling type (fascia or synthetic) by utilizing the Florida State Ambulatory Surgery Database. This database contains only ambulatory procedures, which likely limits their study to synthetic slings. Notably, they did not demonstrate a decline in implantation of synthetic mesh for SUI in the year after the October 2008 FDA Public Health Notification.

One strength of our study is that by choosing dates between 2007 and 2013, it is inclusive of both of the FDA Public Health Notifications $s^{5,6}$ with one full clinical year prior to the initial Public Health Notification and two full years of clinical data following the second one. Only one other study ${ }^{13}$ has attempted to evaluate changes in surgical practice for SUI and $\mathrm{POP}$ in response to these notifications. The authors compared the number of SUI and POP surgeries, including revisions, in the 6 months before and after the 2011 Public Health Notification using the State Ambulatory Surgery
Databases from California, South Carolina, Illinois, and Maryland. These databases are limited to information about practice patterns in ambulatory surgery centers. Our study is complementary to this one in that it highlights the changes in practice patterns at academic tertiary care centers. Other strengths of our study include the large number of procedures performed, the high volume academic surgeons, and the cross-sectional sampling of tertiary care referral centers across multiple geographic regions in the United States inclusive of inpatient and outpatient surgeries.

Taking our results into consideration in conjunction with the findings of Polland et al., the data appears to suggest that the Food and Drug Administration Safety Communications of 2008 and 2011 may potentially have had an effect on the management of stress urinary incontinence. Both studies observed a decline in the utilization of synthetic mesh slings for treatment of SUI in their respective settings, although neither trend was statistically significant. ${ }^{13}$ While the total

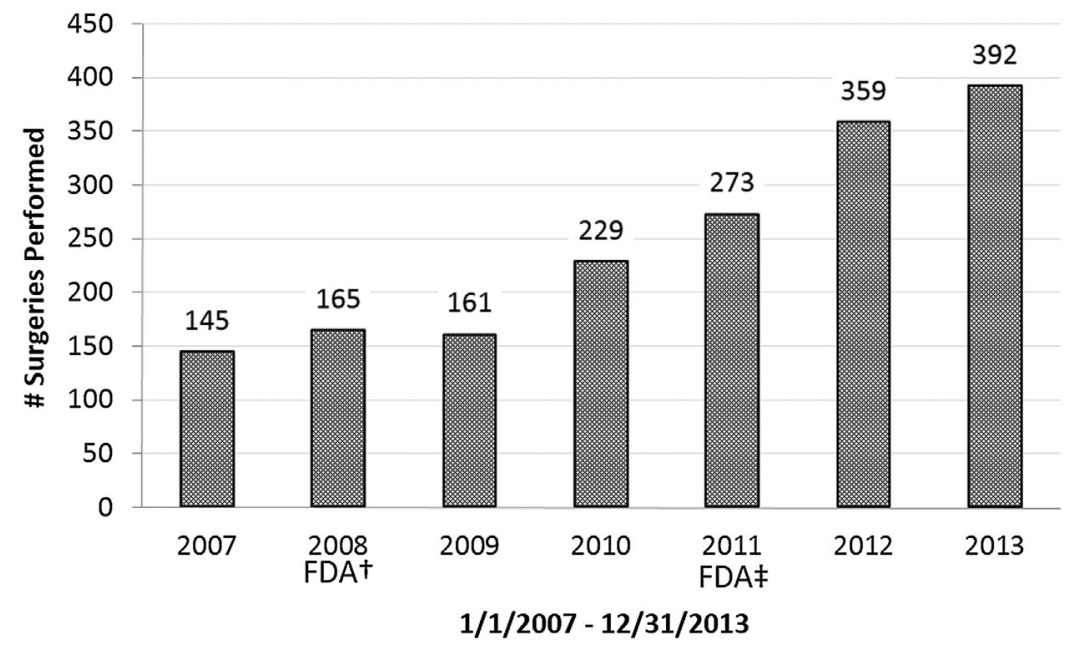

Fig. 4. Trend in mesh revision surgery: urethral intervention for sling. CPT codes for urethrolysis, transvaginal, secondary, open, including cystourethroscopy (53500), and removal or revision of sling for stress incontinence (57287). FDA $\dagger$-October 2008 Public Health Notification and Safety Communication, FDA $\$-J u l y ~ 2011$ UPDATE to Public Health Notification and Safety Communication. 
number of surgical interventions for SUI has remained stable over the study period, there has been a significant increase in the utilization of AFPVS as a percentage of total SUI intervention. In their 2015 abstract, Khan et al. independently confirm this increase in utilization of AFPVS at New York University Langone Medical Center. ${ }^{14}$ Similar to Polland et al., we found a significant increase in sling revision procedures since the FDA Public Health Notifications $(P<0.001$ and $P=0.00045$, respectively). When we stratified the revision data by center, three of the centers had a statistically significant increase in revision surgeries, and three of them had an increase in revision surgeries that was not statistically significant. Two centers had a decrease in mesh revision surgeries, one of which was statistically significant (Lahey Hospital \& Medical Center). However, the significance of this trend is diminished by the fact that the number of revision cases they perform annually is quite low, with a low of 10 in 2011 and a high of 16 in 2007. These observed trends could also be related to changes in referral patterns, an increased awareness and recognition of potential mesh complications, an actual increase in mesh complications, or other factors. This review is not necessarily representative of community and non-tertiary center practice where autologous fascial slings are much less commonly performed. The increased use of AFPVS at these centers is likely due to a combination of factors, not the least of which are the complexity of the patients seen at tertiary centers and the bias seen by these FPMRS tertiary care surgeons due to common evaluation and treatment of mesh related complications.

Limitations of this study include potential regional variations in practice patterns and coding practice as well as bias of tertiary care facilities and associated referral patterns. Fluctuations in the number of providers at each center may have occurred over the course of the study, although each center had at least one individual surgeon throughout the entire study period. By exclusively investigating procedures performed at academic centers, we introduced the possibility of bias via center effect, whereby one academic center could skew the observed trends for the aggregate multicenter data and limit the generalizability of the observed trends. In an attempt to identify and minimize this center effect, we also stratified and observed trends by center. Due to the size of the multi-institutional study we analyzed only case volume using CPT codes for procedures. This method of data collection has limitations that include the inability to identify what the indications for mesh removal were. Additionally, the time-lapse between original surgery and re-intervention is unknown. The CPT codes themselves have certain limitations. For example, the code for urethrolysis (53500) cannot differentiate midurethral slings from bladder neck slings, nor can it clearly determine whether the revision was done due to mesh or because a fascial sling was too tight. Further studies that necessitate approval from the Institutional Review Board can address these issues. Despite these limitations, it is clear that FPMRS practice patterns have changed over the last decade.

\section{CONCLUSIONS}

While the total surgical volume for SUI intervention has remained stable over the last 7 years, it is evident that there is increased use of AFPVS during this time period. During this time period, there has been a concurrent increase in mesh sling revision surgeries. These findings suggest a potential lasting effect of the FDA Public Health Notifications on practice patterns for treatment of SUI among tertiary care FPMRS providers at academic medical centers, even though the Safety Communications did not specifically warn against the use of synthetic mesh for this indication or report on specific complications for midurethral slings for SUI. This trend is likely the result of multiple factors reflecting the complex medico-legal and regulatory atmospheres as well as evolving patient perceptions of TVM.

\section{AKNOWLEDGMENTS}

We appreciate contributions by the biostatistics department at the Medical University of South Carolina. The project and analysis described in the materials and methods section was supported by Award Number UL1TR000062 from the National Center for Advancing Translational Sciences. The content is solely the responsibility of the authors and does not necessarily represent the official views of the National Institutes of Health.

\section{REFERENCES}

1. Geller EJ, Wu JM. Changing trends in surgery for stress urinary incontinence. Curr Opin Obstet Gynecol 2013;25:404-9.

2. Anger JT, Weinberg AE, Albo ME, et al. Trends in surgical management of stress urinary incontinence among female Medicare beneficiaries. Urology 2009:74:283-7.

3. Johnson Funk M, Levin PJ, Wu JM. Trends in the surgical management of stress urinary incontinence. Obstet Gynecol 2012;119:845-51.

4. US Food and Drug Administration. 510(k) Premarket Notification K974098. Jan 28, 1998. Available from: http://www.accessdata.fda.gov/scripts/cdrh/ cfdocs/cfPMN/pmn.cfm?ID = K974098

5. US Food and Drug Administration. FDA Public Health Notification: Serious Complications Associated with Transvaginal Placement of Surgical Mesh in Repair of Pelvic Organ Prolapse and Stress Urinary Incontinence. October 2008. Available from: http://www.fda.gov/MedicalDevices/Safety/ AlertsandNotices/PublicHealthNotifications/ucm061976.htm

6. US Food and Drug Administration. Urogynecologic Surgical Mesh: Update on the Safety and Effectiveness of Transvaginal Placement for Pelvic Organ Prolapse. July 2011. Available from: http://www.fda.gov/downloads/ MedicalDevices/Safety/AlertsandNotices/UCM262760.pdf

7. Miklos JR, Chinthakanan O, Moore RD, et al. The IUGA/ICS classification of synthetic mesh complications in female pelvic floor reconstructive surgery: A multicenter study. Int Urogynecol J 2016;27:933-8.

8. Rice NT, Hu Y, Slaughter JC, et al. Pelvic mesh complications in women before and after the 2011 FDA public health notification. Female Pelvic Med Reconstr Surg 2013;19:333-8.

9. Koski ME, Chamberlain J, Rosoff J, et al. Patient perception of transvaginal mesh and the media. Urology 2014;84:575-82.

10. Younger A, Rac G, Clemens JO, et al. Pelvic organ prolapse surgery trends in academic female pelvic medicine and reconstructive surgery urology practice in the setting of the food and drug administration public health notifications. Urology 2016;91:46-51.

11. Rogo-Gupta L, Litwin MS, Saigal CS, et al. Urologic Diseases in America Project. Trends in the surgical management of stress urinary incontinence among female Medicare beneficiaries, 2002-2007. Urology 2013;82:38-42.

12. Suskind AM, Kaufman SR, Dunn RL, et al. Population-based trends in ambulatory surgery for urinary incontinence. Int Urogynecol J 2013;24: 207-11.

13. Polland A, Meckel K, Trop CS. Incidence of placement and removal or revision of transvaginal mesh for pelvic organ prolapse and stress urinary incontinence in the ambulatory setting before and after the 2011 food and drug administration notification. Urology Practice 2015;2:160-4.

14. Khan A, Rosenblum N, Brucker B, et al. Management of patients seeking care for stress urinary incontinece over the past four years. J Urol Supplement. 2015;193:e1030. 


\section{APPENDIX 1}

Common Procedural Technology (CPT) Codes Referenced

20920 Fascia lata graft; by stripper

29022 Fascia lata graft; by incision and area exposure, complex or sheet

20926 Tissue grafts, other (e.g., paratenon, fat, dermis)

53500 Urethrolysis, transvaginal, secondary, open, including cystourethroscopy (e.g., postsurgical obstruction, scarring)

57287 Removal or revision of sling for stress incontinence (e.g., fascia or synthetic)

57288 Sling operation for stress incontinence (e.g., fascia or synthetic) 